\title{
Uncertainty Evaluation for Measurement result of Pavement Permeability Coefficient
}

\author{
Lu Liu', a , Genqiang Jing ${ }^{2, b}$ and Hongbo Guo ${ }^{3, c}$ \\ ${ }^{1}$ Research Institute of Highway Ministry of Transport, People's Republic of China,8Xitucheng \\ Road, Beijing 100088 \\ ${ }^{2}$ Research Institute of Highway Ministry of Transport, People's Republic of China,8Xitucheng \\ Road, Beijing 100088 \\ ${ }^{3}$ Research Institute of Highway Ministry of Transport, People's Republic of China,8Xitucheng \\ Road, Beijing 100088
}

aliulu@ncmerb.com,bgq.jing@rioh.com,chb.guo@rioh.com

\begin{abstract}
Keywords: Pavement Permeability Coefficient; Permeability Test; Measurement Result; Uncertainty; Asphalt Pavement

Abstract. The measurement result representation must contain both the value to be measured and the measurement uncertainty associated with the value, which is complete and meaningful. When the pavement permeability coefficient is measured on the road, the measurement results are given to the pavement seepage coefficient, and the description of the measurement uncertainty is lacking. The main contents of this paper include: the test method and calculation method of pavement permeability coefficient, the sources of the uncertainty of the measurement result, and the black box mathematical model of uncertainty evaluation. In this paper, the method of uncertainty evaluation for measurement results of pavement permeability coefficient is studied. The components of the uncertainty were evaluated, and the combined uncertainty and the expanded uncertainty were calculated by combining the experimental example of permeability test. The study of this paper provides a method for the uncertainty of the measurement results of pavement seepage coefficient, and provides the basis for the engineering technician to determine whether a measurement result is available.
\end{abstract}

\section{Introduction}

Water permeability of pavement is the ability of the asphalt pavement surface water through the gap within a certain thickness of surface infiltration, which affects the service life of pavement and safety after the rain. Pavement permeability coefficient which obtained by infiltration test is the water infiltration of certain road area per unit time under certain initial static pressure head. Pavement permeability coefficient is used in evaluating the permeability performance asphalt pavement in present engineering quality evaluation. Pavement permeability coefficient is a key index to measure drainage property of asphalt pavement and bituminous mixture ${ }^{[1]}$.

For a long time, pavement permeability coefficient as an important evaluation index of the asphalt pavement status and maintenance level can't be able to adopt advanced automatic testing instrument for on-site inspection, like roughness, rut, slip resistance testing operation. Manual test is the only method nowadays. JJF 1059-1059“Evaluation and Expression of Uncertainty in Measurement"[2] provides that for any measurement with a numerical requirement, in principle, the measurement result and measurement uncertainty of the result should be given at the same time, otherwise the measurement result is incomplete. Uncertainty is an indicator of measurement level, and the basis for judging the measurement result. The reliability and validity for pavement permeability coefficient which is obtained by manual testing is still questioned by many parties in the process of testing evaluation. In order to ensure the validity of the measurement result, while the measurement result of pavement permeability coefficient is given, the uncertainty of measurement must be evaluated and the uncertainty of the measurement result must be given to prove that the measurement results are credible. In this paper, based on the analysis of pavement water seepage form, the test method and the measurement result of pavement permeability coefficient are studied. The sources of uncertainty are evaluated. The combined uncertainty and expanded uncertainty are calculated. Through the 
analysis of the sources of uncertainty for the measurement result of pavement permeability coefficient and the evaluation of the uncertainty, the reliability of the measurement results is ensured and the comparability between the measurement results of different pavement permeability coefficients is enhanced.

\section{Testing method of pavement permeability coefficient}

Current specification JTG E60-2008 "Field Test Methods of Subgrade and Pavement for Highway Engineering" ${ }^{[3]}$ stipulates test instruments, test methods for pavement permeability coefficient.

Apparatus of pavement permeability coefficient as the test instrument has an upper water measuring cylinder made of transparent organic glass, and the volume is $600 \mathrm{ml}$. There are scales on the measuring cylinder, and there are thick lines between $100 \mathrm{ml}$ and $500 \mathrm{ml}$. There is a $\Phi 10 \mathrm{~mm}$ thin tube connected to the measuring cylinder and the base, and there is a switch in the middle. The measuring cylinder is fixed on the base by a bracket. Under the base there is a round hole with an inner diameter of $150 \mathrm{~mm}$ and an outer diameter of $220 \mathrm{~mm}$. Two stainless steel rings with internal diameter of $\varnothing 160 \mathrm{~mm}$ are used to stabilize the instrument, each of them is approximately $5 \mathrm{~kg}$.

When testing, firstly we should seal the gap between the instrument and the road surface in order to prevent the water out from the gap. Then assemble the balancing weight and close the switch. After that, fill the measuring cylinder with water and open the switch, so that the water flows down to drain the air out from the bottom of the instrument. When the water level in the measuring cylinder decreases more slowly, press the instrument using both hands to drain all the bubbles in the bottom of the instrument. Then close the switch and refill the measuring cylinder with water. Open the switch. When the water level decreases to $100 \mathrm{~mL}$, start the stopwatch time immediately. Record the scale every $60 \mathrm{~s}$ until the water level decreases to $500 \mathrm{~mL}$. During the test, if the water seeps from the base, you should move to the dry road surface nearby to re-operate. If the water level decreases too slowly, we just need to record 3 times scales from the time that the water level down to $100 \mathrm{~mL}$. If the water level decreases too quickly and reaches the $500 \mathrm{~mL}$ scale in less than 3 minutes, you should record the reaching time. If the water level decreases to a certain extent and remained unchanged, it indicates that the pavement is basically no water seepage or no water seepage. According to the above steps, choose five measuring points in the same test road section to test pavement permeability coefficient, and the average value is taken as the measurement result.

\section{Computing method of pavement permeability coefficient}

When Darcy's law is met, there are two basic test methods used to test pavement permeability coefficient of the material, which are constant head permeability test and variable head permeability test. The constant head permeability test is usually applied to the strongly permeable material and the variable head permeability test is generally applicable to the weakly permeable material. Suitable test methods for the permeability test of various materials are shown in Table 1.

Tab.1 Applicable permeability test methods for different material

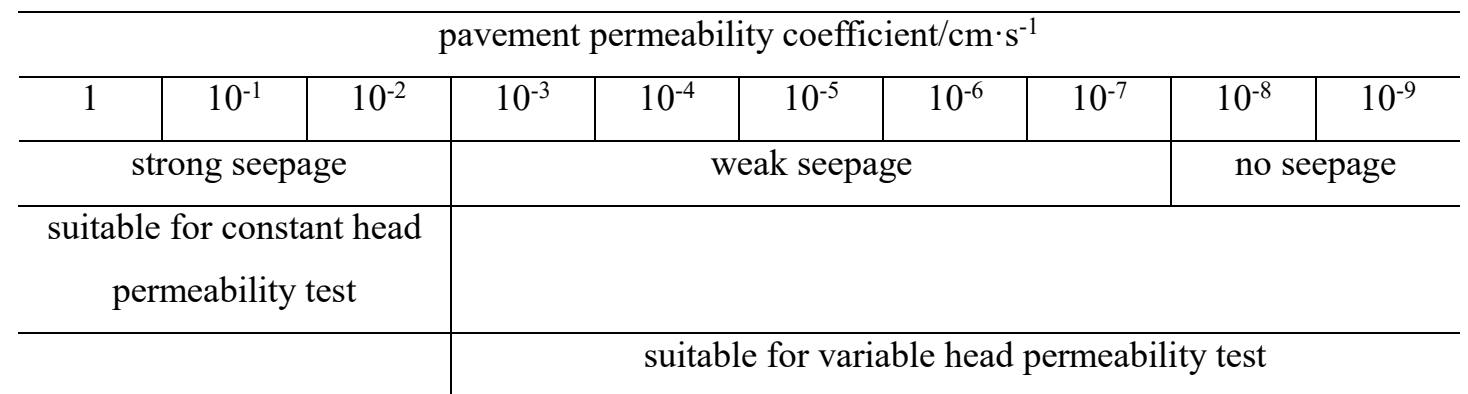

For asphalt mixtures, according to the survey result by G.W.Maupin et al, pavement permeability coefficient of the most asphalt mixtures is in $10^{-3} \sim 10^{-6} \mathrm{~cm} / \mathrm{s}$, and only a handful of pavement permeability coefficient is bigger than $10^{-2} \mathrm{~cm} / \mathrm{s}$. NCAT also recommended a critical pavement permeability coefficient for coarse and fine graded asphalt mixture. Although these data aren't got 
from the standard variable head permeability test, they still indicate the magnitude of pavement permeability coefficient of the asphalt mixture ${ }^{[4,5]}$. According to table 1 , variable head permeability test should be applied to dense asphalt mixtures. The pavement permeability coefficient of variable water head is calculated as follows.

$$
K=\frac{a \cdot L}{A \cdot t} \ln \frac{h_{1}}{h_{2}}
$$

Where, $K$ is pavement permeability coefficient $(\mathrm{cm} / \mathrm{s}), a$ is cross sectional area of water pipe $(\mathrm{cm})$, $I$ is specimen height $(\mathrm{cm}), A$ is specimen cross sectional area $\left(\mathrm{cm}^{2}\right), t$ is the test time $(\mathrm{s}), h_{l}$ is the water head when starting time $(\mathrm{cm}), h_{2}$ is the water head when ending time $(\mathrm{cm})$.

The permeability method stipulated by JTG E60-2008 "Field Test Methods of Subgrade and Pavement for Highway Engineering" is actually a kind of variable head permeability test. The permeability method stipulated by JTG E60-2008 "roadbed road test procedures" is actually a kind of variable head permeability test. When calculating, use the time which water surface decreasing from $100 \mathrm{~mL}$ to $500 \mathrm{~mL}$ for the standard, if the seepage time is too long, the water capacity downing through the measuring cylinder during 3 minutes can be used to calculate. The pavement permeability coefficient is calculated as follows.

$$
C_{w}=\frac{v_{2}-v_{1}}{t_{2}-t_{1}} \cdot 60
$$

Where, $C_{w}$ is pavement permeability coefficient $(\mathrm{mL} / \mathrm{min}), V_{l}$ is the first time water capacity $(\mathrm{mL})$, usually for $100 \mathrm{~mL}, V_{2}$ is the second time water capacity $(\mathrm{mL})$, usually for $500 \mathrm{~mL}, t_{1}$ is the first timing time(s), $t_{1}$ is the second timing time(s).

\section{The mathematical model of uncertainty evaluation}

In uncertainty evaluation, a suitable mathematical model is the key to assess whether the uncertainty evaluation is reasonable. GUM department pointed out that when evaluating uncertainty, the mathematical expression between the measured variable $Y$ and input variables $X_{i}$ need to be given. Also, this expression should include all influencing factors, which have significant influences on the uncertainty of measurement results, including correction values and correction factors. Establishing mathematical model and looking for the sources of influencing the measurement uncertainty should be executed synchronously and iteratively. A good mathematical model should be able to satisfy the following conditions ${ }^{[6]}$ :

(1) The mathematical model should include all inputs that have significant influences on the measurement uncertainty and shouldn't miss any measurement uncertainty components that have significant influences on the measurement uncertainty.

(2) Do not repeat the calculation of any measurement uncertainty components that have significant influences on the measurement uncertainty.

(3) When the input is different, sometimes the mathematical model can be written in different forms. The correlation between the inputs may also be different. At this point, in general the appropriate input should be chosen in order to avoid dealing with troublesome correlations.

According to the above principles, based on the calculation formula of pavement permeability coefficient, the preliminary uncertainty mathematical model is derived as follows:

$$
C_{w}=\frac{\Delta v}{\Delta t}
$$

Where, $\Delta V$ is the measurement result of water seepage $(\mathrm{mL}), \Delta t$ is the measurement result of seepage time $(\mathrm{s})$.

During the measurement process, there are many factors that affect the measurement results, mainly from the following aspects ${ }^{[7]}$ : (a) The definition for the measured is incomplete. (b)The repetition of the definition for the measured is not ideal. (c)Sampling representativeness is not enough. (d) The lack of knowledge about the impact of environmental conditions on the measurement or the 
imperfect measurement of environmental conditions. (e) Offset of personnel readings for analog instruments. (f) Limitations of metrological performance of measuring instruments, such as the maximum allowable error limits. (g) The standard value provided by the measurement standard or standard substance is inaccurate. (h) The referenced constant or other parameter value is not accurate. (i) Approximations and assumptions in measurement methods and measurement processes. (j) Under the same conditions, the changes in repeated observed measurement value. The source of the measurement uncertainty must be analyzed according to the actual measurement. In the analysis, in addition to the definition of uncertainty, the measuring instruments, measurement environment, surveyors, test methods and so on should be fully considered.

According to the influence factors of the above measurement results, the sources of the uncertainty of the permeability measurement results on the same test road section on the same asphalt pavement are analyzed, including the following factors:

(1) The uncertainty introduced by repeatability measurements

Repeatability is the variation in measurements taken by a single person or instrument on the same item, under the same conditions, and in a short period of time. The repeatability coefficient is a precision measure which represents the value below which the absolute difference between two repeated measurement results may be expected to lie with a probability of $95 \%$. The standard deviation under repeatability conditions is part of precision and accuracy. The uncertainty introduced by repeatability measurements is the measurement uncertainty components of the measurement result of pavement permeability coefficient.

(2) The uncertainty introduced by capacity measurement

According to the calculation formula of pavement permeability coefficient, analysis combined with the (e) and (f) sources of uncertainty. Measuring capacity using water measuring cylinder, so the uncertainty from the water measuring cylinder resolution and artificial reading is the measurement uncertainty components of the measurement result of pavement permeability coefficient.

(3) The uncertainty introduced by time measurement

Time measurement is similar to capacity measurement. Measuring capacity using water measuring cylinder, so the uncertainty from the stopwatch resolution and artificial reading is the measurement uncertainty components of the measurement result of pavement permeability coefficient.

(4) The uncertainty introduced by apparatus of pavement permeability coefficient

Analyzing combined with the (f) source of uncertainty. apparatus of pavement permeability coefficient measuring performance limitation is usually given by measuring department through the calibration certificate.

Through the above analysis of the measurement uncertainty components, the calculation model of the uncertainty of the measurement results of pavement permeability coefficient is further improved, and all the uncertainty components are reflected in the mathematical model. The uncertainties introduced by the capacity measurement and the uncertainty introduced by the time measurement have been reflected in the mathematical model, but the uncertainty introduced by the repeatability measurement and the uncertainty introduced by apparatus of pavement permeability coefficient can be dealt by the black box model. Therefore, the optimized mathematical model is shown in formula (4) .

$$
C_{w}=\frac{\Delta v}{\Delta t} \delta_{1} \delta_{2}
$$

Where, $\delta_{1}$ is the uncertainty introduced by repeatability measurements, $\delta_{2}$ is the uncertainty introduced by apparatus of pavement permeability coefficient.

\section{Evaluation and calculation of uncertainty}

For each source of uncertainty, it is not necessary to distinguish whether the influence is random or systematic, but to consider how to estimate the standard deviation. The uncertainty component that the standard deviation can be calculated from the measured data is the A-class standard uncertainty component, and the remaining components belong to the B-class standard uncertainty component. 


\section{The relative uncertainty component introduced by repeatability measurements $u_{r \delta 1}$}

The qualified inspectors use the calibrated apparatus of pavement permeability coefficient choosing five measuring points to determine pavement permeability coefficient in the same test section, then take the average value as the measurement results.

Tab.2 Five determined pavement permeability coefficient in the same test section

$$
\text { unit: } \mathrm{mL} / \mathrm{min}
$$

\begin{tabular}{c|c|c|c|c|c|c}
\hline points number & 1 & 2 & 3 & 4 & 5 & average \\
\hline determined value & 392.30 & 392.30 & 391.38 & 391.38 & 390.47 & 391.97 \\
\hline
\end{tabular}

The uncertainty component introduced by the repeatability measurement is evaluated by independently assessing the data obtained by the measurement, which should be evaluated using the A-class evaluable method. The Bessel method is the most commonly used A-class evaluation method to input uncertainty. However, when using the Bessel formula, the number of measurements $n$ can' $t$ be too small, otherwise the resulting standard uncertainty $u\left(x_{k}\right)=s\left(x_{k}\right)$ in addition to itself there will be a large uncertainty, there are system errors associated with the number of measurements $n$. The $n$ is smaller; the system error is greater. So, how much should the measurement number $\mathrm{n}$ be, depending on the specific circumstances of the measurement. JJF1033-2008 "Rule for the Examination of Measurement Standard" $[8]$ provides when measuring the repeatability of the measurement standard, the number of measurements $n \geq 10$ is required. At the same time, the relevant information is also clear: When the A class standard uncertainty component in the measurement results of the synthetic standard uncertainty plays a major role, $\mathrm{n}$ should not be too small, preferably not less than 10 . According to above principle, in the actual pavement permeability coefficient test experiment, actually selecting five points to test does not meet the requirements of the Bessel method. Therefore, the experimental standard deviation $s\left(x_{k}\right)$ of the measurement results $x_{k}$ of pavement permeability coefficient given by range-method which is suitable for the number of measurements is small is used to evaluate approximately. Computation formula is shown as follows:

$$
\left(x_{k}\right)=s\left(x_{k}\right)=R / C=1.83 / 2.33=0.79 \mathrm{~mL} / \mathrm{min}
$$

Where, $R$ is range, the difference between the maximum and minimum value in the measurement results. $C$ is coefficient of range, through the look-up table.

In the pavement permeability coefficient obtained in the actual measurement, the average value of the five measurements is used as the best estimate of the measurement result. At this time, the experimental standard deviation $s(\bar{x})$ of the mean $\bar{x}$ is measured by a single test Standard deviation $s\left(x_{k}\right)$.The standard uncertainty introduced by capacity measurement is calculated the following:

$$
u_{\delta 1}=s(\bar{x})=u\left(x_{k}\right) / \sqrt{n}=0.79 / \sqrt{5}=0.35 \mathrm{~mL} / \mathrm{min}
$$

Relative standard uncertainty is calculated the following:

$$
u_{r \delta 1}=0.35 / 391.97=0.09 \%
$$

\section{The relative standard uncertainty introduced by capacity measurement $u_{r \Delta v}$}

The standard uncertainty introduced by the capacity measurement is based on the B-class evaluation method, and the standard deviation is estimated by scientific judgment with all available information. Usually in accordance with the relevant information or experience, determine possible value range $(-a, a)$ is measured. Assuming the probability distribution of the measured value, the confidence factor (or coverage factor) $\mathrm{k}$ is estimated from the probability distribution and the required confidence level $\mathrm{P}$, the B-class standard uncertainty can be calculated as follows:

$$
u_{B}=\frac{a}{k}
$$

Where, $a$ is the half width of the measured value interval, $k$ is confidence coefficient (coverage 
factor).

The result of pavement permeability coefficient is calculated by the difference between the two times the amount of water seepage. The calculating formula is $\Delta V=V_{2}-V_{1}$, measurement uncertainty components introduced by $V_{2}$ and $V_{1}$ are respectively calculated.

(1) The uncertainty $\left(u_{2}\right)$ introduced by water seepage $\left(V_{2}\right)$ of the second timing

The uncertainty introduced by water seepage of the second timing includes two measurement uncertainty components: one is introduced by the least definition of water measuring cylinder, the other one is introduced by the human eye reading delay.

a. The uncertainty introduced by the least definition of water measuring cylinder $\left(u_{21}\right)$

The smallest scale of water measuring cylinder is $2 \mathrm{ml}$, so the uncertainty introduced by the least definition of water measuring cylinder is

$$
u_{21}=a / k=2 / 2 \sqrt{3}=0.58 \mathrm{~mL}
$$

b. The uncertainty introduced by the human eye reading delay $\left(u_{22}\right)$

In the process of permeability test, press the stopwatch, at the same time read the value of the water level in the measuring cylinder. Human reading level is about $10 \mathrm{~ms}$ delay time, the uncertainty introduced by the human eye reading level delay is

$$
u_{22}=a / k=391.97 / 60 \times 10 \times 10^{-3}=0.065 \mathrm{~mL}
$$

c. The calculation of uncertainty $\left(u_{2}\right)$

This part of the calculation is the actual calculation of combined uncertainty. Combined uncertainty is synthesized from the standard uncertainty components, regardless of the standard uncertainty components are evaluated by class A or class B. When the measurement $Y$ is determined by other quantity $X_{1}, X_{2}, \ldots, X_{N}$, the measuring result of measurement y is:

$$
y=f\left(x_{1}, x_{2}, \cdots x_{N}\right)
$$

The combined uncertainty $u_{c}(y)$ of measuring result is calculated according to the formula (6).

$$
u_{c}(y)=\sqrt{\sum_{i=1}^{N}\left[\frac{\partial f}{\partial x_{i}}\right]^{2} u^{2}\left(x_{i}\right)+2 \sum_{i=1}^{N-1} \sum_{i=1}^{N} \frac{\partial f}{\partial x_{i}} \frac{\partial f}{\partial x_{j}} r\left(x_{i}, x_{j}\right) u\left(x_{i}\right) u\left(x_{j}\right)}
$$

Where, $y$ is the estimated value of the output, that is, the measuring result of measurement. $x_{i}, x_{j}$ is estimated value of input quantity, $i \neq j . N$ is the number of inputs. $\frac{\partial f}{\partial x_{i}}, \frac{\partial f}{\partial x_{j}}$ is partial derivative, known as sensitivity coefficient, which can also be represented by the symbols $c_{i}, c_{j} . u\left(x_{i}\right)$ is standard uncertainty of input quantity $\left(x_{i}\right) . u\left(x_{j}\right)$ is standard uncertainty of input quantity $\left(x_{j}\right)$; $r\left(x_{i}, x_{j}\right)$ is estimated value of correlation coefficient for input quantity $x_{i}, x_{j}$. $r\left(x_{i}, x_{j}\right) \mu\left(x_{i}\right) \mu\left(x_{j}\right)=u x_{i}, x_{j}$ is estimated value of covariance for input quantity $x_{i}, x_{j}$. The formula(6)is known as uncertainty propagation law(or known as uncertainty transitive law), which is a general formula.

There is no correlation between the uncertainties in introduced by the least definition of water measuring cylinder and the human eye reading delay, so $r\left(x_{i}, x_{j}\right)=0$. The combined uncertainty is

$$
u_{2}=\sqrt{u_{21}^{2}+u_{22}^{2}}=\sqrt{0.58^{2}+0.065^{2}}=0.58 \mathrm{~mL}
$$

(2) The uncertainty $\left(u_{1}\right)$ introduced by water seepage $\left(V_{1}\right)$ of the first timing

In a similar way, the uncertainty $\left(u_{1}\right)$ introduced by water seepage $\left(V_{1}\right)$ of the second timing includes two measurement uncertainty components.

$$
u_{1}=u_{2}=0.58 \mathrm{~mL}
$$


(3) The relative uncertainty introduced by capacity measurement $u_{r \Delta v}$

Due to there is strong positive correlation for the water seepage between first timing and second timing, the correlation coefficient is $r\left(x_{i}, x_{j}\right)=1$. The uncertainty propagation law formula can be simplified as the algebraic sum of the uncertainty components. The standard uncertainty introduced by $\Delta \mathrm{V}$ is

$$
u_{r \Delta v}=u_{1}+u_{2}=0.58+0.58=1.16 \mathrm{~mL}
$$

Take $\Delta V=400 \mathrm{~mL}$, the relative uncertainty introduced by capacity measurement is

$$
u_{r \Delta v}=\frac{1.16}{400} \times 100 \%=0.9 \%
$$

\section{The relative uncertainty introduced by time measurement $\left({ }^{u_{r \Delta t}}\right)$}

$$
\Delta t=t_{2}-t_{1}
$$

The uncertainty introduced by the first timing is mainly introduced by the minimum resolution of stopwatch. The minimum scale of stopwatch is $1 \mathrm{~s}$. So the uncertainty introduced by the minimum resolution is

$$
u_{t 21}=a / k=1 / 2 \sqrt{3}=0.28 \mathrm{~s}
$$

Due to there is strong positive correlation for the time between first timing time and second timing time, the correlation coefficient is 1 . So the relative uncertainty introduced by time measurement is

$$
u_{\Delta t}=0.28+0.28=0.56 \mathrm{~s}
$$

The computing process of uncertainty is similar to capacity measurement. The time interval is $\Delta t=61.23 \mathrm{~s}$, so the relative uncertainty introduced by time measurement is

$$
u_{r \Delta t}=\frac{0.56}{61.23} \times 100 \%=0.91 \%
$$

\section{The relative uncertainty introduced by apparatus of pavement permeability coefficient $\left({ }^{u_{r \delta 2}}\right)$}

The apparatus of pavement permeability coefficient is calibrated by metrology technical organization. The relative expanded uncertainty shown in calibration certificate $U_{r}=3.1 \%(k=2)$. Therefore, the relative uncertainty introduced by the apparatus of pavement permeability coefficient is $u_{r \delta 2}$

$$
u_{r \delta 2}=3.1 \% / 2=1.55 \%
$$

\section{The calculation of relative expanded uncertainty}

The extended uncertainty is obtained by multiplying the combined uncertainty by the coverage factor. Due to the unrelated correlation between the uncertainty components, the relative combined uncertainty is

$$
\begin{aligned}
u_{r} & =\sqrt{u_{r \delta 1}^{2}+u_{r \Delta v}^{2}+u_{r \Delta t}^{2}+u_{r \delta 2}^{2}} \\
& =\sqrt{0.09 \%^{2}+0.29 \%^{2}+0.91 \%^{2}+1.55 \%^{2}}=1.82 \%
\end{aligned}
$$

The value of the coverage factor $(\mathrm{k})$ is selected based on the confidence level required for the interval $y \pm U$ determined by $U=k u_{c} . k$ value is generally taken 2 to 3 . In the engineering survey, generally take $k=2$. Therefore, the relative expanded uncertainty of pavement permeability coefficient is

$$
U_{r}=1.82 \% \times 2=3.64 \%
$$

The report of measuring result for pavement permeability coefficient is

$$
C_{w}=391.97 \mathrm{~mL} / \min , U_{r}=3.64 \%(k=2)
$$

\section{Conclusions}

Through the analysis of test method and calculation method for the pavement permeability coefficient, the sources of uncertainty mainly come from repeatability measurement, capacity 
measurement, time measurement and apparatus of pavement permeability coefficient limitations.

The mathematical model of uncertainty evaluation is established, and the uncertainty evaluation method for measurement results of pavement permeability coefficient is developed, which can be used for other permeability test.

The components of the uncertainty were evaluated, and the combined uncertainty and the expanded uncertainty were calculated by combining the experimental example of permeability test.

Uncertainty, as part of the measurement results for pavement permeability coefficient, characterizes the quality of the measurement results and increases the comparability of measurement results for different pavement seepage tests.

\section{References}

[1] DAN Han-cheng, LI Liang, HE Yao-chao, et al. Study on permeability test of asphalt pavement[J]. Journal of Highway and Transportation Research and Development, 2008, 26(8):16-21. (In Chinese)

[2] JJF 1059-2012, Evaluation and Expression of Uncertainty in Measurement [S]. (In Chinese )

[3] JTG E60-2008, Field Test methods of subgrade and pavement forhighway engineering[S]. （In Chinese )

[4] CHEN Jing, SUN Peng-tao, LI Fu-pu, et al. Study on permeability coefficient of asphalt mixture[J]. Journal of Highway and Transportation Research and Development, 2006, 23(1): 5-8. (In Chinese)

[5]LIU Qing-quan, CAO Dong-wei. Research on material composition and performance of porous asphalt pavement[J].Journal Material in Civil Engineering, ACSE, 2009, 21(4):135-140.

[6] NI-yucai. Evaluation of practical measurement uncertainty[S], China Metrology Publishing House

[7] International Organization for Standardization, Guide to the Expression of Uncertainty in Measurement[S] ,1993. (In Chinese)

[8] JJF 1033-2008 " Rule for the Examination of Measurement Standard”.[S] （In Chinese ) 